\title{
НОВЫЙ СПОСОБ ТАМПОНАДЫ ТАЗА ПРИ ПРОДОЛЖАЮЩЕМСЯ ВНУТРИТАЗОВОМ КРОВОТЕЧЕНИИ
}

\author{
К. А. Егиазарян ${ }^{1}$, Д. И. Гордиенко ${ }^{1}$, Д. А. Старчик², А. М. Лыско ${ }^{1 凶}$ \\ 1 Российский национальный исследовательский медицинский университет имени Н. И. Пирогова, Москва, Россия \\ ${ }^{2}$ Северо-Западный государственный медицинский университет имени И. И. Мечникова, Санкт-Петербург, Россия
}

\begin{abstract}
Нестабильные повреждения тазового кольца продолжают оставаться одной из наиболее частых причин летального исхода у пациентов с сочетанной травмой, а существующие способы имеют ряд осложнений и противопоказаний. Нами описан успешный клинический случай остановки внутритазового кровотечения у пациента с множественными травмами, в котором с целью объединения преимуществ малой инвазивности ангиоэмболизации, простоты исполнения и воздействия на основную причину кровопотери тампонады таза применен новый способ внутритазовой остановки кровотечения при помощи баллонных устройств.
\end{abstract}

Ключевые слова: сочетанная травма, множественная травма, политравма, переломы костей таза, внутритазовое кровотечение, тампонада таза

Информация о вкладе авторов: К. А. Егиазарян, Д. И. Гордиенко - организация и планирование исследования; Д. А. Старчик - планирование исследования, выполнение анатомического исследования; А. М. Лыско - анализ литературы, сбор, анализ, интерпретация данных, выполнение оперативного вмешательства.

Соблюдение этических стандартов: исследование одобрено этическим комитетом городской клинической больницы № 1 имени Н. И. Пирогова (протокол № 5 от 28 мая 2018 г.) и этическим комитетом РНИМУ имени Н. И. Пирогова (протокол № 170 от 18 декабря 2017 г.).

$\triangle$ Для корреспонденции: Артём Михайлович Лыско

ул. Хачатуряна, д. 12, корп. 3, г. Москва, 127562; ArtLysko@gmail.com

Статья получена: 03.03.2019 Статья принята к печати: 16.04.2019 Опубликована онлайн: 29.04.2019

DOI: $10.24075 /$ vrgmu.2019.031

\section{THE NEW METHOD OF PELVIC PACKING AGAINST CONTINUING INTRAPELVIC BLEEDING RESULTING FROM THE UNSTABLE PELVIC RING FRACTURES}

Egiazaryan $\mathrm{KA}^{1}$, Gordienko DI' , Starchik DA², Lysko AM'1 ${ }^{1}$

${ }^{1}$ Pirogov Russian National Research Medical University, Moscow, Russia

${ }^{2}$ Mechnikov North-Western State Medical University, Saint-Peterburg, Russia

Unstable pelvic ring fractures are one of the common causes of death of patients with concomitant injuries. The existing methods applied to treat such conditions can cause a number of complications and have contraindications. We have described a successful clinical case of intrapelvic hemorrhage arrest in a patient with multiple injuries. in this case, we applied the new method combining minimally invasive angioembolization and easily applicable and effective balloon tamponade.

Keywords: concomitant injury, multiple injury, polytrauma, intrapelvic bleeding, pelvic packing

Author contribution: Egiazaryan KA, Gordienko DI — study organization and planning; Starchik DA — study planning, anatomical examination; Lysko AM — literature analysis, data collection, analysis, interpretation, surgery.

Compliance with ethical standards: the study was approved by the Ethics Committee of Pirogov City Clinical Hospital № 1 (minutes № 5 of May 28, 2018) and Ethics Committee of Pirogov Russian National Research Medical University (minutes № 170 of December 18, 2017).

Correspondence should be addressed: Artyom M. Lysko

Khachaturiana 12-3, Moscow, 127562; ArtLysko@gmail.com

Received: 03.03.2019 Accepted: 16.04.2019 Published online: 29.04.2019

DOI: 10.24075/brsmu.2019.031

Высокая актуальность проблемы остановки внутритазового кровотечения, развившегося вследствие нестабильных повреждений тазового кольца, обусловлена достаточно высокой частотой летальных исходов и значимыми медицинскими и социальными последствиями [1]. Исходя из последних данных повреждения тазового кольца более чем в 25\% случаев встречаются в составе сочетанной и множественной травм [2-4]. До 60\% данных повреждений имеют летальные последствия, каждый третий случай из которых - следствие неконтролируемого внутритазового кровотечения [5-9].

За многие годы в поисках решения проблемы был разработан ряд методов внутритазовой остановки кровотечения, таких как ангиоэмболизация, тампонада таза и REBOA (Resuscitative Endovascular Balloon Occlusion of the Aorta) [10-16]. Каждый из них уникален и имеет ряд своих положительных и отрицательных аспектов. Тампонаду таза применяют при более часто встречаемом венозном кровотечении, она инвазивнее других методов, что приводит к большей кровопотере и инфекционным осложнениям. Ангиоэмболизация, в свою очередь, может быть осложнена ишемическими повреждениями, ведущими к некрозам и развитию внутричерепного кровоизлияния [17-22]. С целью учета таких преимуществ, как малоинвазивность ангиоэмболизации, простота исполнения и эффективность воздействия на основную причину кровопотери тампонады таза, нами разработан новый способ остановки внутритазового кровотечения. Следует отметить, что в основу идеи взят распространенный в акушерстве и гинекологии метод баллонной тампонады матки, применяемый при неконтролируемых внутриматочных кровотечениях [23]. 


\section{Описание клинического случая}

Пациент С. 26 лет, строитель по специальности, пострадал в результате падения на бетонную плиту с высоты 3 этажа (около 12 м). На догоспитальном этапе пациенту начата инфузионная и обезболивающая терапия. При поступлении пациент был интубирован, выполнены катетеризация вен, мочевого пузыря, адекватная инфузионная, обезболивающая терапия. Показатели артериального давления (АД) пациента составляли 107/70 мм рт. ст. при пульсе 113 уд./мин с нормальным темпом диуреза. Состояние по шкале комы Глазго оценили в 9 баллов. По данным лабораторных анализов показатели гемоглобина и гематокрита пациента находились в пределах нормы при выраженном лейкоцитозе (до 29,9 • 109 кл./л), дефиците оснований (9,8 ммоль/л) и уровне лактата 4,8 ммоль/л. Ультразвуковое исследование при поступлении показало отсутствие жидкости в плевральной и брюшной полостях и незначительное ее количество в полости малого таза. Учитывая стабильность гемодинамики и наличие клинических признаков механической нестабильности тазового кольца на момент поступления в стационар, пациенту выполнили наложение тазового бандажа, после чего он был отправлен на ПАН КТ (компьютерную томографию всех отделов организма). В ходе исследования в составе сочетанной травмы у пациента выявили повреждение тазового кольца типа С1 по классификации АО (Arbeitsgemeinschaft für Osteosynthesefragen)/OTA (Orthopaedic Trauma Association) (рис. 1). Кроме того обнаружены закрытая черепно-мозговая травма, ушиб теменной доли головного мозга справа, переломы костей носа, стенок левой орбиты, закрытый перелом большого бугорка правого плеча с незначительным смещением отломков, вывих в суставе Лисфранка правой стопы, гематома мошонки (по шкале ISS = 29 баллов).

В ходе КТ-исследования произошла резкая дестабилизация гемодинамики пациента с падением давления до 60/30 мм рт. ст., с учащением пульса до 150 уд./мин; начата вазопрессорная поддержка раствором норадреналина 0,2\% (0,3 мкг/кг/мин), пациент был экстренно взят в операционную для стабилизации переднего полукольца таза надцетабулярным аппаратом наружной фиксации, наложения С-рамы Ганца на заднее полукольцо. Экстренные мероприятия по стабилизации тазового кольца привели к подъему показателей АД до 85/40 мм рт. ст. и урежению пульса до 130 уд./мин на фоне продолжающейся вазопрессорной поддержки. Учитывая сохраняющуюся гемодинамическую нестабильность в течение 15 мин после механической стабилизации тазового кольца, мы приняли решение о выполнении малоинвазивной баллонной тампонады таза следующим способом: в положении пациента на спине под местной анестезией (инфильтрация мягких тканей в области доступа растворами местных анестетиков) непосредственно над лонным сочленением по передней срединной линии тела произвели разрез-прокол кожи размерами до 2-3 см. Подкожную клетчатку развели тупым способом, достигая пирамидальной мышцы, волокна которой также развели тупым способом. Через данное отверстие ввели троакар со стилетом в предпузырное клетчаточное пространство (пространство Ретциуса). Далее троакар со стилетом направили паравезикально в направлении левого или правого крестцово-подвздошного сочленения в соответствующее боковое клетчаточное пространство (рис. 2). После удаления стилета по троакару в область повреждения ввели баллонное устройство для остановки внутриматочного кровотечения (баллон Жуковского) (ООО «Гинамед»; Россия), состоящее из пластикового остова длиной до 25 см, диаметром до 8 мм, и резинового баллона размерами до 11,5 см. Троакар удален. Затем процедуру повторили в противоположном направлении. Баллонные устройства были одновременно заполнены стерильным физиологическим раствором. В ходе заполнения было отмечено постепенное нарастание отчетливого сопротивления на поршне шприца Жане с последующим возникновением его обратного хода после заполнения баллонных устройств до 110 мл, что было расценено нами как признак достаточного объема инфляции. С целью контроля за положением и целостностью баллонных устройств выполнили интраоперационные рентгенограммы таза на аппарате Phillips BV Endura

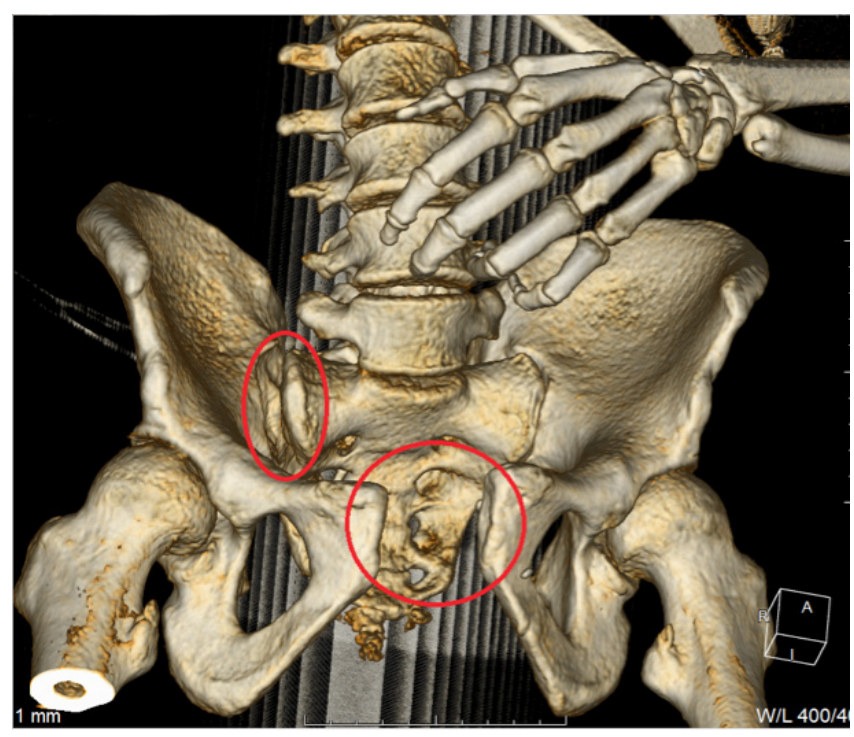

Рис. 1. 3D-КТ-модель повреждения тазового кольца: красным показань разрывы правого крестцово-подвздошного сочленения и лонного сочленения

Таблица. Динамика показателей состояния пациента до и после проведения оперативного вмешательства

\begin{tabular}{|l|c|c|c|}
\hline \multicolumn{1}{|c|}{ Показатели/время } & Поступление & $\begin{array}{c}\text { Через час после оперативного } \\
\text { вмешательства }\end{array}$ & Конец первых суток с поступления \\
\hline АД (мм рт. ст.) & $60 / 30$ & $110 / 47$ & $115 / 70$ \\
\hline ЧСС (уд./мин) & 150 & 115 & $100-110$ \\
\hline Гемоглобин (г/л) & 149 & 128 & 92 \\
\hline Гематокрит (\%) & 44,2 & 38,4 & 27,2 \\
\hline Лейкоцитоз (109/л) & 29,9 & 33 & 2,8 \\
\hline Лактат (ммоль/л) & 4,8 & 7,4 & $-2,7$ \\
\hline Дефицит оснований (ммоль/л) & $-9,8$ & -14 & \\
\hline
\end{tabular}


(Нидерланды) (рис. 3). Концы баллонных устройств вывели на кожу и подшили, рану послойно ушили (рис. 4). Выполнение малоинвазивной внутритазовой тампонады по заявленному способу привело к подъему показателей АД до 110/47 мм рт. ст. с урежением пульса до 115 уд./мин. В раннем послеоперационном периоде пациента перевели в отделение реанимации. В течение первых суток показатели гемодинамики стабилизировались до 115/70 мм рт. ст. с пульсом 100-110 уд./мин, к концу первых суток постепенно отменили вазопрессорную поддержку. Динамика показателей состояния пациента представлена в таблице. Темпы диуреза у пациента оставались на достаточном уровне, признаки кровотечения отсутствовали. Через 48 часов с момента поступления на фоне стабильных гемодинамических и лабораторных показателей было принято решение о тестовой дефляции баллонных устройств на 50 мл и последующем наблюдении за пациентом. С учетом стабильности состояния пациента

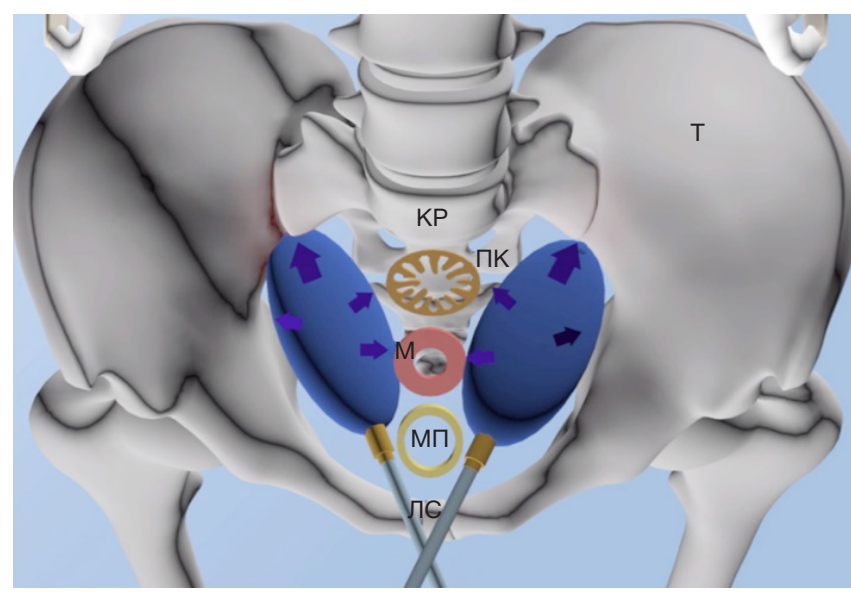

Рис. 2. Схематичное изображение направления установки баллонных устройств в полости малого таза. МП - мочевой пузырь, М - матка, ПК - прямая кишка, КР - крестец, Т - безымянная кость, ЛС лобковый симфиз. Синим указаны баллоны, установленные в латеральные пространства малого таза

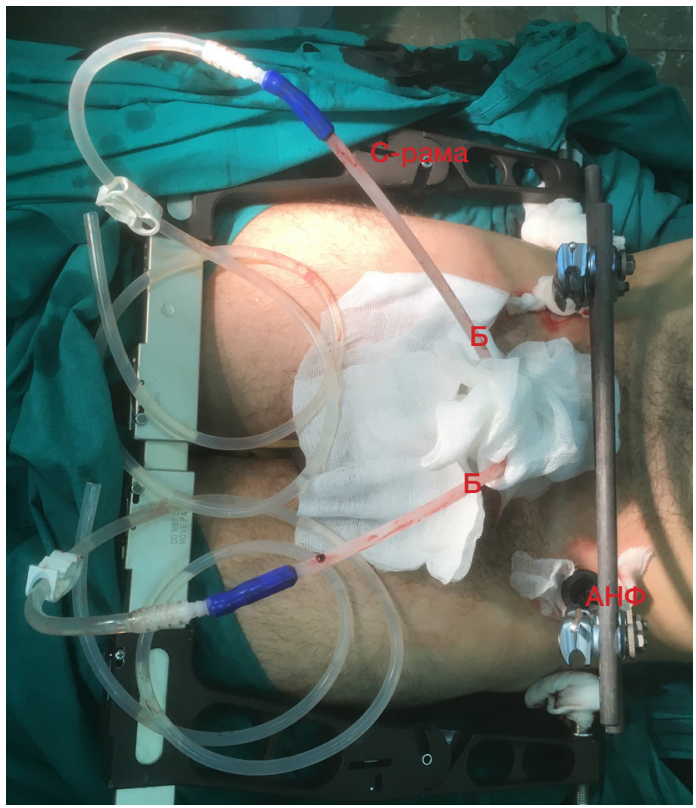

Рис. 4. Внешний вид тазовой области пациента с нестабильным повреждением тазового кольца и развитием внутритазового кровотечения после стабилизации аппаратами наружной фиксации и установки баллонных устройств в полость малого таза: С-рама — Рама Ганца на заднее полукольцо, АНФ — аппарат наружной фиксации на переднее полукольцо, Б - баллонные устройства на третьи сутки с момента поступления принято решение о демонтаже С-рамы и стабилизации задних отделов таза винтом, а также удалении баллонных устройств из полости малого таза (рис. 5). На 5-е сутки с момента поступления пациент был переведен в профильное отделение травматологии и ортопедии для дальнейшего лечения с ежедневным контролем послеоперационной раны: отмечено ее заживление первичным натяжением без признаков воспаления и патологического отделяемого, что позволило на 10-е сутки выполнить окончательную стабилизацию тазового кольца и на 16-е сутки выписать пациента на амбулаторное долечивание (рис. 6, 7).

\section{Обсуждение клинического случая}

Международное сообщество регулярно разрабатывает рекомендации по ведению пациентов с нестабильными повреждениями тазового кольца. Согласно наиболее

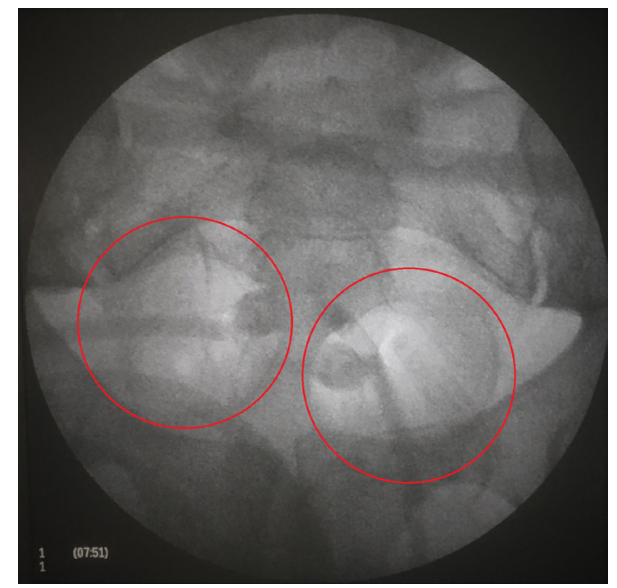

Рис. 3. Интраоперационная рентгенограмма таза у пациента с нестабильным повреждением тазового кольца и развитием внутритазового кровотечения после стабилизации аппаратами наружной фиксации и установки баллонных устройств в полость малого таза: красным показаны контуры баллонных устройств в полости малого таза

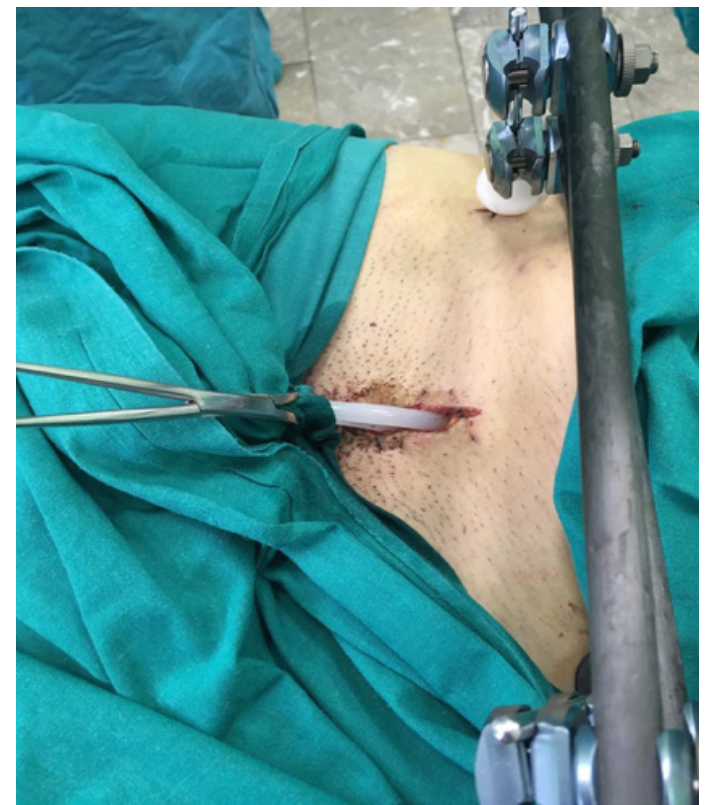

Рис. 5. Внешний вид раны пациента с выступающими трубками баллонных устройств непосредственно перед удалением на третьи сутки. Рана без признаков воспаления, по ходу баллонных устройств отсутствует патологическое отделяемое. В правой половине фотографии аппарат наружной фиксации на переднее полукольцо таза 
актуальным из них, первым этапом следует выполнять механическую стабилизацию тазового кольца аппаратами наружной фиксации с последующим переходом на внутритазовые методы остановки, предпочтительнее из которых тампонада таза, так как венозное кровотечение встречается в 8 из 10 случаев против 2 из 10 в случае артериального кровотечения [24]. Если после выполнения тампонады таза гемодинамика пациента остается нестабильной, рекомендовано выполнение транскатетерной ангиоэмболизации кровоточащих сосудов [24]. Следуя данным рекомендациям, наличие у пациента одновременно механической и гемодинамической нестабильности служит показанием к последовательному выполнению механической стабилизации тазового кольца и тампонады таза, как и в представленном выше клиническом примере, где вмешательство оказалось достаточным для стабилизации гемодинамики. Преимуществом разработанного нами способа является контролируемая инфляция баллонных устройств, позволяющая выполнять поэтапную дефляцию уже после 24 ч с момента установки под четким контролем стабильности гемодинамических показателей. Данный способ также облегчает определение источника кровотечения при выполнении транскатетерной ангиоэмболизации. Не менее важным аспектом является безопасность использования стерильного и нейтрального по отношению к средам организма физиологического раствора: в случае прорыва баллонного устройства он окажется безвредным, что позволит снизить потенциальный риск развития осложнений.

Следует отметить, что мировая тенденция к выбору малоинвазивных решений в сфере медицинских вмешательств не обошла стороной тазовую хирургию. В 2015 г. был описан клинический случай выполнения тампонады малого таза за счет катетеризации мочевого пузыря его инфляцией физиологическим раствором в объеме 500-600 мл [25]. Абсолютным противопоказанием к выполнению данной методики является подтверждение нарушения целостности мочевыводящих путей. Выполнение тампонады наполненным мочевым пузырем в раннем послеоперационном периоде позволило добиться стабилизации гемодинамики пациентки. Однако, в связи с нарастанием признаков почечной недостаточности, пришлось провести десрляцию мочевого пузыря, после чего гемодинамика пациентки дестабилизировалась, что привело к выполнению лапаротомии. В декабре 2016 г. в результате проведения экспериментальной работы на животных, был предложен новый способ малоинвазивной тампонады внутритазового кровотечения баллонным устройством, расположенным в предпузырном пространстве [26]. Эфффективность метода изучали в сравнении с группой животных, которым была выполнена стандартная тампонада, и контрольной группой без использования внутритазовой остановки кровотечения. В ходе эксперимента была доказана эффективность малоинвазивной баллонной тампонады. Однако следует отметить, что расположение баллонного устройства в передних отделах малого таза может спровоцировать смещение фрлотирующих костных фрагментов переднего полукольца, не поддающихся внешней фиксации, выдавливание внутренних органов в задние отделы таза с возможной персорацией последних костными отломками, а также разрыв и повреждение уретры при чрезмерном отдавливании мочевого пузыря. По мнению авторского коллектива, симметричное расположение баллонных устройств в боковых клетчаточных пространствах должно предотвратить развитие подобных осложнений.

\section{ВЫВОДЫ}

Разработанный нами способ тампонады таза можно считать перспективным ввиду малой инвазивности, ограниченного объема вызываемых повреждений и возможности контролировать давление в баллонных устройствах. Коллектив авторов выражает надежду на то, что данный метод найдет свое применение в ведении пациентов с нестабильными повреждениями тазового кольца.

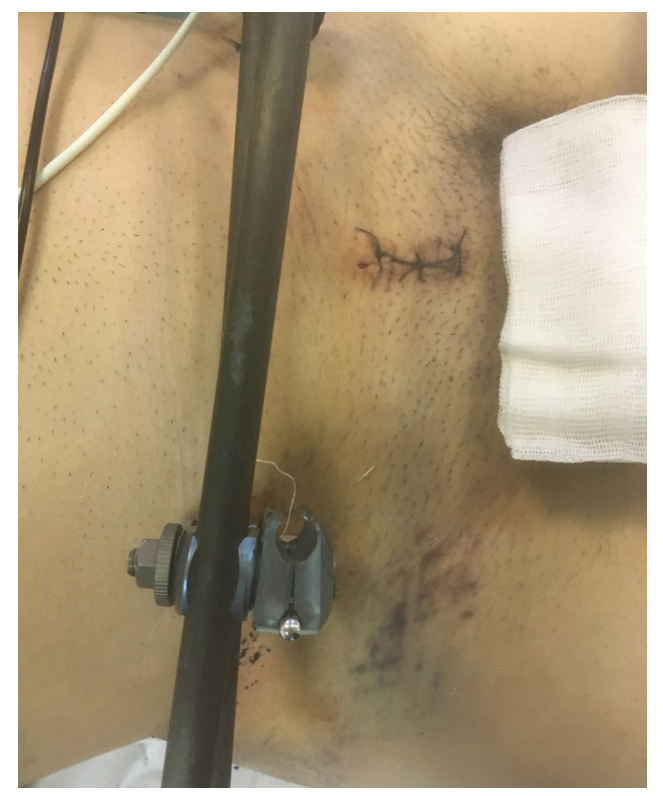

Рис. 6. Вид послеоперационной раны через сутки после удаления баллонных устройств. Послеоперационная рана заживает первичным натяжением без признаков воспаления и патологического отделяемого, швы состоятельны. В левой половине фотографии аппарат наружной фиксации на переднее полукольцо таза

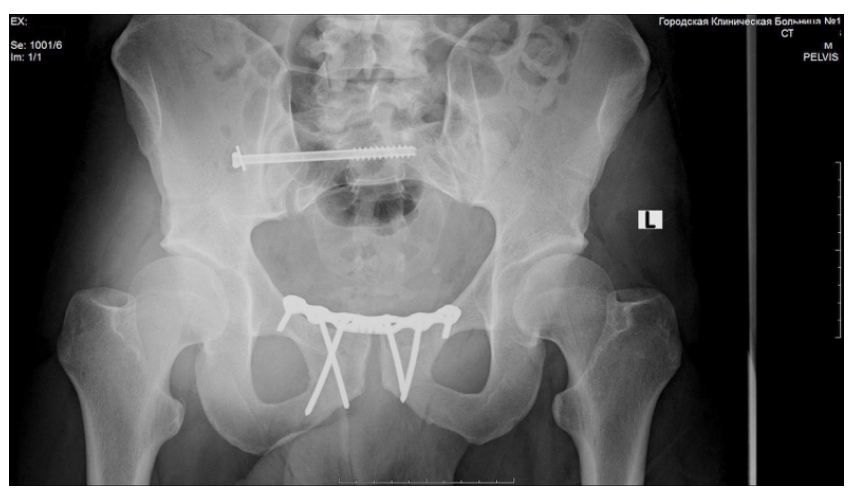

Рис. 7. Рентгенограмма таза пациента после окончательной стабилизации тазового кольца на 10-е сутки: фиксация правого крестцово-подвздошного сочленения спонгиозным винтом с частичной нарезкой и шайбой, лонного сочленения пластиной и винтами 
1. Смоляр А. Н. Диагностика и лечение травматических забрюшинных кровоизлияний [диссертация]. М., 2012-272.

2. Burkhardt M, Kristen A, Culemann U, Koehler D, Histing T, Holstein $J$ et al. Pelvic fracture in multiple trauma: Are we still upto-date with massive fluid resuscitation? Injury. 2014; (45): 70-5.

3. Esmer E, Esmer E, Derst P, Schulz M, Siekmann H, Delank K Einfluss der externen Beckenstabilisierung bei hämodynamisch instabilen Beckenfrakturen. Der Unfallchirurg. 2015; 120 (4): 312-9.

4. Wohlrath B, Trentzsch H, Hoffmann R, Kremer M, SchmidtHorlohè K, Schweigkofler U. Preclinical and clinical treatment of instable pelvic injuries: Results of an online survey. Der Unfallchirurg. 2014; 119 (9): 755-62. In German.

5. Holcomb J, del Junco D, Fox E, Wade C, Cohen M, Schreiber M, et al. The Prospective, Observational, Multicenter, Major Trauma Transfusion (PROMMTT) Study. JAMA Surgery. 2013; 48 (2): 27.

6. Guerado E, Bertrand M, Valdes L, Cruz E, Cano J. Resuscitation of Polytrauma Patients: The Management of Massive Skeletal Bleeding. The Open Orthopaedics Journal. 2015; 9 (1): 283-95.

7. Costantini T, Coimbra R, Holcomb J, Podbielski J, Catalano R, Blackburn A, et al. Current management of hemorrhage from severe pelvic fractures. Journal of Trauma and Acute Care Surgery. 2016; 80 (5): 717-25.

8. Burlew C, Moore E, Stahel P, Geddes A, Wagenaar A, Pieracci F, et al. Preperitoneal pelvic packing reduces mortality in patients with life-threatening hemorrhage due to unstable pelvic fractures. Journal of Trauma and Acute Care Surgery. 2017; 82 (2): 233-42.

9. Tesoriero R, Bruns B, Narayan M, Dubose J, Guliani S, Brenner M, et al. Angiographic embolization for hemorrhage following pelvic fracture. Journal of Trauma and Acute Care Surgery. 2017; 82 (1): 18-26.

10. Файн А. М. Диагностика и лечение тяжелых переломов костей таза у пострадавших с сочетанной и множественной травмой [диссертация]. М., 2017-238.

11. Guerado E, Medina A, Mata M, Galvan J, Bertrand M. Protocols for massive blood transfusion: when and why, and potential complications. European Journal of Trauma and Emergency Surgery. 2015; 42 (3): 283-95

12. Margolies M, Ring E, Waltman A, Kerr W, Baum S. Arteriography in the Management of Hemorrhage from Pelvic Fractures. New England Journal of Medicine. 1972; 287 (7): 317-21.

13. Pohlemann T, Gansslen A, Bosch U, Tscherne H. The Technique of Packing for Control of Hemorrhage in Complex Pelvic Fractures. Techniques in Orthopaedics. 1994; 9 (4): 267-70.

14. Smith W, Moore E, Osborn P, Agudelo J, Morgan S, Parekh A, et al. Retroperitoneal Packing as a Resuscitation Technique for Hemodynamically Unstable Patients with Pelvic Fractures: Report of Two Representative Cases and a Description of Technique. The Journal of Trauma: Injury, Infection, and Critical Care. 2005; 59 (6): 1510-4.

15. Hughes C. W. Use of an intra-aortic balloon catheter tamponade for controlling intra-abdominal hemorrhage in man. Surgery. 1954; 36 (1): 65-8.

16. DuBose J, Scalea T, Brenner M, Skiada D, Inaba K, Cannon J et al. The AAST prospective Aortic Occlusion for Resuscitation in Trauma and Acute Care Surgery (AORTA) registry. Journal of Trauma and Acute Care Surgery. 2016; 81 (3): 409-19.

17. Matityahu A, Marmor M, Elson J, Lieber C, Rogalski G, Lin C, et al. Acute Complications of Patients With Pelvic Fractures After Pelvic Angiographic Embolization. Clinical Orthopaedics and Related Research. 2013; 471 (9): 2906-11.

18. Salcedo E, Brown I, Corwin M, Galante J. Pelvic angioembolization in trauma - Indications and outcomes. International Journal of Surgery. 2016; (33): 231-6.

19. Rudloff M, Triantafillou K. Management of Pelvic Ring Injuries in Unstable Patients. Orthopedic Clinics of North America. 2016; 47 (3): 551-63.

20. Saito N, Matsumoto H, Yagi T, Hara Y, Hayashida K, Motomura T, et al. Evaluation of the safety and feasibility of resuscitative endovascular balloon occlusion of the aorta. Journal of Trauma and Acute Care Surgery. 2015; 78 (5): 897-904.

21. Davidson A, Russo R, Reva V, Brenner M, Moore L, Ball C, et al. The pitfalls of resuscitative endovascular balloon occlusion of the aorta. Journal of Trauma and Acute Care Surgery. 2018; 84 (1): 192-202.

22. Uchino H, Tamura N, Echigoya R, Ikegami T, Fukuoka T. "REBOA" Is it Really Safe? A Case with Massive Intracranial Hemorrhage Possibly due to Endovascular Balloon Occlusion of the Aorta (REBOA). American Journal of Case Reports. 2016; 17: 810-3.

23. Charoenkwan K. Effective use of the Bakri postpartum balloon for posthysterectomy pelvic floor hemorrhage. American Journal of Obstetrics and Gynecology. 2014; 210 (6): 586.

24. Coccolini F, Stahel P, Montori G, Biffl W, Horer T, Catena F, et al. Pelvic trauma: WSES classification and guidelines. World Journal of Emergency Surgery. 2017; 12 (1): 5.

25. Huang S, Vohora A, Russ M, Mathew J, Johnny C, Stevens J, et al. Delaying urinary catheter insertion in the reception and resuscitation of blunt multitrauma and using a full bladder to tamponade pelvic bleeding. Injury. 2015; 46 (6): 1081-3.

26. Sokol K, Black G, Willey S, Song M, Marko S, Eckert M, et al. Preperitoneal balloon tamponade for lethal closed retroperitoneal pelvic hemorrhage in a swine model. Journal of Trauma and Acute Care Surgery. 2016; 81 (6): 1046-55.

\section{References}

1. Smoljar AN. Diagnostic and treatment of traumatic retroperitoneal hemorrhages [dissertation]. M., 2012-272. In Russian.

2. Burkhardt M, Kristen A, Culemann U, Koehler D, Histing T, Holstein J, et al. Pelvic fracture in multiple trauma: Are we still upto-date with massive fluid resuscitation? Injury. 2014; (45): 70-5.

3. Esmer E, Esmer E, Derst P, Schulz M, Siekmann H, Delank K. Einfluss der externen Beckenstabilisierung bei hämodynamisch instabilen Beckenfrakturen. Der Unfallchirurg. 2015; 120 (4): 312-9.

4. Wohlrath B, Trentzsch H, Hoffmann R, Kremer M, SchmidtHorlohè K, Schweigkofler U. Preclinical and clinical treatment of instable pelvic injuries: Results of an online survey. Der Unfallchirurg. 2014; 119 (9): 755-62. In German.

5. Holcomb J, del Junco D, Fox E, Wade C, Cohen M, Schreiber M, et al. The Prospective, Observational, Multicenter, Major Trauma Transfusion (PROMMTT) Study. JAMA Surgery. 2013; 48 (2): 27.

6. Guerado E, Bertrand M, Valdes L, Cruz E, Cano J. Resuscitation of Polytrauma Patients: The Management of Massive Skeletal Bleeding. The Open Orthopaedics Journal. 2015; 9 (1): 283-95.

7. Costantini T, Coimbra R, Holcomb J, Podbielski J, Catalano R,

Blackburn A, et al. Current management of hemorrhage from severe pelvic fractures. Journal of Trauma and Acute Care Surgery. 2016; 80 (5): 717-25.

8. Burlew C, Moore E, Stahel P, Geddes A, Wagenaar A, Pieracci F, et al. Preperitoneal pelvic packing reduces mortality in patients with life-threatening hemorrhage due to unstable pelvic fractures. Journal of Trauma and Acute Care Surgery. 2017; 82 (2): 233-42.

9. Tesoriero R, Bruns B, Narayan M, Dubose J, Guliani S, Brenner M, et al. Angiographic embolization for hemorrhage following pelvic fracture. Journal of Trauma and Acute Care Surgery. 2017; 82 (1): 18-26.

10. Fajn AM. Diagnostic and treatment of severe pelvic fractures by patients with concomitant and multiple injuries [dissertation]. M., 2017-238. Russian.

11. Guerado E, Medina A, Mata M, Galvan J, Bertrand M. Protocols for massive blood transfusion: when and why, and potential complications. European Journal of Trauma and Emergency Surgery. 2015; 42 (3): 283-95.

12. Margolies M, Ring E, Waltman A, Kerr W, Baum S. Arteriography 
in the Management of Hemorrhage from Pelvic Fractures. New England Journal of Medicine. 1972; 287 (7): 317-21.

13. Pohlemann T, Gansslen A, Bosch U, Tscherne H. The Technique of Packing for Control of Hemorrhage in Complex Pelvic Fractures. Techniques in Orthopaedics. 1994; 9 (4): 267-70.

14. Smith W, Moore E, Osborn P, Agudelo J, Morgan S, Parekh A, et al. Retroperitoneal Packing as a Resuscitation Technique for Hemodynamically Unstable Patients with Pelvic Fractures: Report of Two Representative Cases and a Description of Technique. The Journal of Trauma: Injury, Infection, and Critical Care. 2005; 59 (6): 1510-4.

15. Hughes C. W. Use of an intra-aortic balloon catheter tamponade for controlling intra-abdominal hemorrhage in man. Surgery. 1954; 36 (1): 65-8.

16. DuBose J, Scalea T, Brenner M, Skiada D, Inaba K, Cannon J, et al. The AAST prospective Aortic Occlusion for Resuscitation in Trauma and Acute Care Surgery (AORTA) registry. Journal of Trauma and Acute Care Surgery. 2016; 81 (3): 409-19.

17. Matityahu A, Marmor M, Elson J, Lieber C, Rogalski G, Lin C et al. Acute Complications of Patients With Pelvic Fractures After Pelvic Angiographic Embolization. Clinical Orthopaedics and Related Research. 2013; 471 (9): 2906-11.

18. Salcedo E, Brown I, Corwin M, Galante J. Pelvic angioembolization in trauma - Indications and outcomes. International Journal of Surgery. 2016; (33): 231-6.

19. Rudloff M, Triantafillou K. Management of Pelvic Ring Injuries in Unstable Patients. Orthopedic Clinics of North America. 2016; 47 (3): 551-63.
20. Saito N, Matsumoto H, Yagi T, Hara Y, Hayashida K, Motomura T, et al. Evaluation of the safety and feasibility of resuscitative endovascular balloon occlusion of the aorta. Journal of Trauma and Acute Care Surgery. 2015; 78 (5): 897-904.

21. Davidson A, Russo R, Reva V, Brenner M, Moore L, Ball C, et al. The pitfalls of resuscitative endovascular balloon occlusion of the aorta. Journal of Trauma and Acute Care Surgery. 2018; 84 (1): 192-202.

22. Uchino H, Tamura N, Echigoya R, Ikegami T, Fukuoka T. "REBOA" Is it Really Safe? A Case with Massive Intracranial Hemorrhage Possibly due to Endovascular Balloon Occlusion of the Aorta (REBOA). American Journal of Case Reports. 2016; (17): 810-3.

23. Charoenkwan K. Effective use of the Bakri postpartum balloon for posthysterectomy pelvic floor hemorrhage. American Journal of Obstetrics and Gynecology. 2014; 210 (6): 586.

24. Coccolini F, Stahel P, Montori G, Biffl W, Horer T, Catena F, et al. Pelvic trauma: WSES classification and guidelines. World Journal of Emergency Surgery. 2017; 12 (1): 5.

25. Huang S, Vohora A, Russ M, Mathew J, Johnny C, Stevens J, et al. Delaying urinary catheter insertion in the reception and resuscitation of blunt multitrauma and using a full bladder to tamponade pelvic bleeding. Injury. 2015; 46 (6): 1081-3.

26. Sokol K, Black G, Willey S, Song M, Marko S, Eckert M, et al. Preperitoneal balloon tamponade for lethal closed retroperitoneal pelvic hemorrhage in a swine model. Journal of Trauma and Acute Care Surgery. 2016; 81 (6): 1046-55. 